\title{
VALIDACIÓN DE UN TEST \\ PARA VERIFICAR SI EL ALUMNO \\ POSEE CONCEPCIONES CIENTÍFICAS \\ SOBRE CALOR, TEMPERATURA \\ Y ENERGÍA INTERNA*
}

\author{
LANG DA SIL VEIRA, F. y MOREIRA, M. A. \\ Instituto de Física, UFRGS. Caixa Postal, 15051, Campus. 91501-970 Porto Alegre, RS, Brasil.
}

\begin{abstract}
SUMMARY
This study is a continuation of the task of validating a paper and pencil test to check whether the student has scientific conceptions about heat, temperature and internal energy in which we are working since 1989. The third version of this instrument is presented here. Previous versions (Silveira, Moreira and Axt, 1990, 1991) were modified after an analysis of internal consistency carried out with additional data provided by 168 students of introductory physics at UFRGS. The present version is constituted by 23 items, which might be divided into three groups, each one corresponding to one of the three concepts: heat, temperature and internal energy. This division is based on a logical content analysis as well as on an empirical analysis of students' responses.
\end{abstract}

\section{INTRODUCCIÓN}

La razón de construir y validar tests escritos para verificar, específicamente, si los alumnos poseen concepciones cientificas sobre determinado tópico se basa en la hipótesis de que esa información es muy relevante para ta enseñanza y su obtención no es trivial. Hoy se recono$c e$, independientemente del referencial teórico adoptado, que el conocimiento previo de los alumnos, sea éste científico o no, tiene gran influencia en el aprendizaje de nuevos conocimientos. Existe también una cierta aceptación generalizada de que la entrevista clínica es la mejor manera de detectar el verdadero conocimiento previo de un aprendiz. Pero la entrevista clínica no es viable para la práctica diaria en el aula, pues es preciso mucha experiencia y tiempo.

Por esta causa, la salida que nos pareció viable fue la confección de tests escritos, a partir de indicadores obtenidos con entrevistas, y su validación a través de sucesivas aplicaciones.

En este sentido ya construimos y validamos instrumentos de este tipo en las áreas de mecánica y de electricidad. En esta comunicación presentamos la tercera versión de un test para verificar si el alumno posee concepciones científicas sobre calor, temperatura y energía interna.

Escogimos estos tres conceptos, ya que, según el análisis conceptual que hicimos sobre termodinámica, son éstos, juntamente con la entropía, los conceptos claves de esta área. Dado que nos pareció excesivo enfocar cuatro conceptos en un único test, dejamos fuera la entropía.

Por otro lado, aquí no hacemos referencias a investigaciones ya realizadas en torno a esos conceptos, dado que nuestro objetivo no es el de comparar nuestros resultados con los de otros autores. Lo que pretendemos es relatar el proceso de validación de un instrumento para detectar si el alumno tiene concepciones alternativas en ese área.

\section{EL TEST}

El test que presentamos en el anexo es la tercera versión ya que las versiones anteriores fueron modificadas des- 
pués de realizar análisis de consistencia interna, cuya base son las respuestas de más de un centenar de alumnos (Silveira, Moreira y Axt, 1990 y 1991). Esta última versión está constituida por 25 ítems; los ítems 7 y 17 fucron eliminados en el análisis de consistencia interna que presentamos más adelante, quedando finalmente 23 items.

Los îtems poseen tres afirmaciones, identificadas por los números romanos I, II y III. Se ofrecen 7 alternativas de respuesta cn cada f́tem (véanse instruciones en el anexo). Esas 7 alternativas permiten que quien responde opte por una de las afirmaciones, por cualquier combinación de dos afirmaciones o por las tres al mismo tiempo.

Los contenidos del test son los conceptos de calor, temperatura y energía interna. Dentro de las alternativas de respuesta siempre existe una que es compatible con las concepciones científicas sobre tales conceptos; las otras alternativas intentan que quien responde exprese concepciones alternativas.

La forma de cuantificar la respuesta que un determinado sujeto da en cada ítem tiene el objetivo principal de detectar si posee o no la concepción científica; en caso negativo, no se pretende detectar cuall o cuáles son las concepciones alternativas que él posee. A excepción de los ítems 16 y 24 , esta cuantificación es efectuada atribuyendo la puntuación de uno si la alternativa escogida fuese la que es compatible con la concepción científica, y la puntuación de cero en cualquier otra alternativa. Los f́tems 16 y 24 , que son los únicos que tienen como alternativa correcta dos afirmaciones (afirmaciones II y III), son cuantificados de la siguiente manera: la puntuación de uno para la alternativa $\mathrm{F}$; la puntuación de 0,5 para la alternativa $\mathrm{B}$ o $\mathrm{C}$; la puntuación de cero

Tabla 1

Distribución de frecuencias por ítem a través de las alternativas.

\begin{tabular}{|c|c|c|c|c|c|c|c|c|}
\hline \multirow{2}{*}{ Item } & \multicolumn{8}{|c|}{ Alternativa } \\
\hline & A & B & $\mathrm{C}$ & $\mathrm{D}$ & $\mathrm{E}$ & $\mathrm{F}$ & $G$ & Omisiones \\
\hline 1 & 22 & 0 & $131 *$ & 0 & 13 & 2 & 0 & 0 \\
\hline 2 & 29 & $136 *$ & 1 & 0 & 0 & 2 & 0 & 0 \\
\hline 3 & $54^{* t}$ & 2 & 52 & 3 & 51 & 2 & 3 & 1 \\
\hline 4 & 25 & $70^{*}$ & 23 & 19 & 14 & 4 & 12 & 1 \\
\hline 5 & 52 & $104 *$ & 9 & 2 & 1 & 0 & 0 & 0 \\
\hline 6 & $103^{k}$ & 21 & 44 & 0 & 0 & 0 & 0 & 0 \\
\hline 7 & 14 & 31 & $121 *$ & I & j & 0 & 0 & 0 \\
\hline 8 & $123^{*}=$ & 1 & 37 & 1 & 1 & 2 & 3 & 0 \\
\hline 9 & 13 & 28 & $126^{*}$ & 0 & 0 & 0 & 0 & i \\
\hline 10 & 8 & $88 *$ & 0 & 72 & 0 & 0 & 0 & 0 \\
\hline 11 & $130^{*}$ & 0 & 38 & 0 & 0 & 0 & 0 & 0 \\
\hline 12 & 27 & 27 & $107^{*}$ & 6 & 0 & 0 & 0 & 1 \\
\hline 13 & 9 & 49 & $104^{*}$ & 2 & I & 0 & 0 & 3 \\
\hline 14 & $120^{*}$ & 6 & 7 & 17 & 8 & 2 & 7 & 1 \\
\hline 15 & 1 & 23 & $69^{*}$ & 6 & 4 & 34 & 29 & 2 \\
\hline 16 & 13 & 85 & 5 & 29 & 2 & $18^{*}$ & 13 & 3 \\
\hline 17 & $125^{*}$ & 36 & 4 & 0 & 0 & 1 & 0 & 2 \\
\hline 18 & $51^{*}$ & 17 & 9 & 38 & 7 & 10 & 32 & 4 \\
\hline 19 & 32 & $83^{*}$ & 48 & 0 & 1 & 0 & 0 & 4 \\
\hline 20 & 67 & 12 & $65^{*}$ & 5 & 14 & 0 & 0 & 5 \\
\hline 21 & $99^{*}$ & 21 & 35 & 4 & 3 & 0 & 0 & 6 \\
\hline 22 & 17 & $92 *$ & 53 & 1 & 0 & 0 & 0 & 5 \\
\hline 23 & 66 & $79^{*}$ & 5 & 0 & 0 & 9 & 1 & 8 \\
\hline 24 & 31 & 39 & 33 & 0 & 2 & $58 *$ & 0 & 5 \\
\hline 25 & 18 & 34 & $110^{*}$ & I & 0 & 0 & 0 & 5 \\
\hline
\end{tabular}


para cualquier otra alternativa (esta manera de cuantificar determinó un pequeño incremento en la consistencia interna del test en comparación con la forma que atribuía sólo Ia puntuación de cero y uno).

Los ítems pueden ser divididos en tres grupos, cada uno de ellos designando uno de los tres conceptos: calor, temperatura y energía interna. La reunión de los ítems en grupos fue realizada a través de un análisis lógico del contenido de los ítems y de un análisis empírico a partir de las respuestas de 168 alumnos de Fúsica General (ver a continuación el análisis de consistencia interna). Se obtiene una puntuación total (suma de una puntuación en los ítems) en cada uno de los tres grupos de f́tems; cada una de las puntuaciones totales se constituye en una medida del dominio que un individuo tiene de la concepción científica acerca del concepto respectivo.
Es posible también obtener un total único si se suman las puntuaciones en todos los ítems.

\section{ANÁLISIS DE CONSISTENCIA INTERNA}

EI análisis de consistencia interna del test fue llevado a cabo sobre las respuestas de 168 alumnos de Física General de la UFRGS. La mitad de la muestra, aproximadamente, no había estudiado Termodinámica y la otra mitad sí.

Las respuestas de estos alumnos, ítem a ítem, están sintetizadas en la tabla I, donde se presentan las frecuencias absolutas por alternativa. La frecuencia en la alternativa compatible con las concepciones científicas en

\begin{tabular}{|c|c|c|c|c|}
\hline \multirow{2}{*}{ Test } & \multirow{2}{*}{ Ítem } & \multicolumn{3}{|c|}{ EACTOR } \\
\hline & & Calor & Temperatura & $\begin{array}{l}\text { Energía } \\
\text { interna }\end{array}$ \\
\hline Calor & $\begin{array}{l}1 \\
2 \\
3 \\
4 \\
10 \\
14 \\
15 \\
16 \\
18 \\
23\end{array}$ & $\begin{array}{l}0,53 * \\
0,41 * \\
0,61 * \\
0,73 * \\
0,63 * \\
0,48 * \\
0,53 * \\
0,53 * \\
0,58 * \\
0,53 *\end{array}$ & $\begin{array}{l}0,09 \\
0,16+ \\
0,16+ \\
0,32^{*} \\
0,35^{*} \\
0,13 \\
0,20^{*} \\
0,24^{*} \\
0,17+ \\
0,24^{*}\end{array}$ & $\begin{array}{l}0,04 \\
0,21: \\
0,22 \\
0,12 \\
0,13 \\
0,19+ \\
0,06 \\
0,15+ \\
0,11 \\
0,22\end{array}$ \\
\hline Temperatura & $\begin{array}{r}5 \\
8 \\
9 \\
12 \\
13 \\
25\end{array}$ & $\begin{array}{l}0,36^{*} \\
0,22^{*} \\
0,26^{*} \\
0,18 * \\
0,22^{*} \\
0,29^{*}\end{array}$ & $\begin{array}{l}0,62^{*} \\
0,60^{*} \\
0,71^{*} \\
0,79+ \\
0,71^{*} \\
0,63^{*}\end{array}$ & $\begin{array}{l}0,15+ \\
0,03 \\
0,17+ \\
0,09 \\
0,07 \\
0,19+\end{array}$ \\
\hline $\begin{array}{l}\text { Energía } \\
\text { interna }\end{array}$ & $\begin{array}{r}6 \\
11 \\
19 \\
20 \\
21 \\
22 \\
24\end{array}$ & $\begin{array}{l}0,16+ \\
0,07 \\
0,27^{*} \\
0,18+ \\
0,15+ \\
0,05 \\
0,02\end{array}$ & $\begin{array}{l}0,09 \\
-\quad 0,07 \\
0,18+ \\
0,09 \\
0,16+ \\
-\quad 0,01 \\
0,18+\end{array}$ & $\begin{array}{l}0,55^{*} \\
0,46^{*} \\
0,60: \\
0,53^{*} \\
0,56^{*} \\
0,44^{*} \\
0.42\end{array}$ \\
\hline Eliminados & $\begin{array}{r}7 \\
17\end{array}$ & $\begin{array}{l}0,11 \\
0,11\end{array}$ & $\begin{array}{l}0,16 \\
0,09\end{array}$ & $\begin{array}{r}0,01 \\
0,07\end{array}$ \\
\hline
\end{tabular}


cada ítem está indicada con un asterisco. No discutiremos en cada ítem las dificultades presentadas por los alumnos, pues solamente un ítem es un indicador débil de cada concepto. Las inferencias realizadas sobre cada ftem individualmente están sujetas a más errores que las realizadas sobre la puntuación total en el grupo de ítems.

Fue realizado posteriormente un análisis factorial de estas respuestas a través del «método de los grupos múltiples de Thurstone» (Nunnally, 1967; Wherry, 1984). Este método, contrariamente a otros métodos de análisis en los cuales los factores aparecen a posteriori, parte de postular a priori la existencia de factores en grupos de variables (ítems), testando empíricamente (corroborando o no) la inclusión de cada ítem como indicador del concepto identificado a través del análisis lógico del contenido. El método permite concluir también si un ítem es indicador de un concepto diferente de aquel postulado a priori o si debe ser eliminado del test. Cada factor es una combinacion linear de las variables (en general, la suma con igual peso) previamente designadas como indicadores del mismo. Luego, para cada variable, es calculado el coeficiente de correlación de ésta con cada uno de los factores, danco origen a la carga factorial de cada variable en cada factor. En nuestro caso, como postulamos la existencia de tres factores (calor, temperatura y energía interna), tenemos tres cargas factoriales por ítem (Tabla II). Se observa que los ítems 7 y 17 presentam cargas factoriales irrelevantes y sin significancia estadística en cualquiera de los tres factores. Se decidió entonces eliminar estos dos ítems de la versión «definitiva» del test.

Es posible constatar también que diversos ítems poseen una carga factorial razonable en más de un factor, Io que indica que los tres factores no son independientes en la estructura cognitiva de los alumnos. La tabla IH presenta los coeficientes de corretación entre los tres factores, evidenciando un moderado grado de relación entre ellos.

Tabla III

Matriz de correlaciones entre los factores.

\begin{tabular}{|l|c|c|}
\hline \multicolumn{1}{|c|}{ Factor } & Temperatura & Energia interna \\
\hline $\begin{array}{l}\text { Calor } \\
\text { Temperatura }\end{array}$ & $0,37^{*}$ & $0,26^{*}$ \\
$0,17^{*}$
\end{tabular}

* Estadisticamente significativo a nivel $\leq 1 \%$.

Tabla IV

Características de la puntuación en cada test y en todo el test.

\begin{tabular}{|l|c|c|c|c|}
\hline \multicolumn{1}{|c|}{ Test } & Medida & Desviación típica & Coef. alfa & Coef. beta \\
\hline Calor & 5,13 & 2,52 & 0,75 & 0,78 \\
Temperatura & 4,01 & 1,90 & 0,76 & 0,81 \\
Energía interna & 3,96 & 1,67 & 0,52 & 0,60 \\
\hline Total & 13,10 & 4,43 & 0,81 & 0,84 \\
\hline
\end{tabular}

La interdependencia de los factores constituye un apoyo empírico para la confección de una única puntuación total en el test a partir de la suma de las puntuaciones de Ios 23 ftems. Se puede aún observar que algunos ítems, como por ejemplo el ítem 16 , presentan una carga factorial importante en un factor que está en conflicto con el del análisis lógico del contenido. El ítem 16 fue originalmente delineado para verificar si el alumno posee o no el concepto científico de energia interna; sin embargo, su carga factorial más importante se encuentra en el concepto de calor y fue por esta razón que quedo así finalmente clasificado.

El análisis de consistencia interna es complementado a través de las estimaciones de fiabilidad de las 3 puntuaciones totales y de la puntuación total en todo el test. La tabla IV presenta, además del promedio y de la desviáción típica de las puntuaciones totales, las estimaciones 
Tabla V

Estabilidad temporal de las puntuaciones en cada test y de la totalidad.

\begin{tabular}{|l|c|c|c|}
\hline \multirow{2}{*}{ Test } & \multicolumn{2}{|c|}{$\begin{array}{c}\text { Medida geométrica } \\
\text { de las dos aplicaciones }\end{array}$} & $\begin{array}{c}\text { Coeficiente de } \\
\text { correlación entre las dos } \\
\text { aplicaciones }\end{array}$ \\
\cline { 2 - 4 } & A l a & B e t a & $0,76^{*}$ \\
Calor & 0,69 & 0,73 & $0,66^{*}$ \\
Temperatura & 0,60 & 0,67 & $0,55^{*}$ \\
Energía interna & 0,56 & 0,63 & $0,74^{*}$ \\
\hline Total & 0,70 & 0,74 & 0 \\
\hline
\end{tabular}

* Estadísticamente significativo a nivel inferior a $1 \%$.

de fiabilidad realizadas a través de los coeficientes alfa (Cronbach, 1951) y beta (Silveira, 1985). La puntuación total menos fiable se refiere al concepto de energía interna y muestra que, aun en esta version, el test deja que desear en cuanto a ser una medida precisa del dominio de tal concepto en un individuo determinado. Las otras puntuaciones de fiabilidad son razonables, sobre todo si el test fuese utilizado para comparar grupos de individuos respecto a valores promedios (Silveira, 1993).

\section{ESTABILIDAD TEMPORAL DE LAS PUNTUACIONES TOTALES}

En una muestra de 24 alumnos de FIS, 183 de la UFRGS, el test fue aplicado y reaplicado, espaciados aproximadamente en 45 días. Las aplicaciones fueron realizadas después del término de las unidades de Termodinámica en aquella disciplina (los contenidos de la disciplina son: Termodinámica, Ondas Mecánicas y Óptica Geométri-
Se verifica nuevamente que la puntuación total menos confiable se refiere al concepto de energía interna.

ca). Los coeficientes de correlación entre las puntuaciones obtenidas en las dos aplicaciones se constituyen también en estimaciones de fiabilidades de esas puntuaciones (Nunnally, 1967); los coeficientes de consistencia interna (alfa y beta) estiman a partir de presupuestos diferentes. La teoría de la medida piscológica permite suponer que ambas estimaciones (consistencia interna y estabilidad temporal) puedan ser coherentes. De manera más precisa, la media geométrica de los coeficientes de consistencia interna en las dos aplicaciones debe ser semejante al coeficiente de correlación entre las dos aplicaciones. La tabla $\mathrm{V}$ presenta los tesuitados obtenidos.

\section{VALIDEZ PREDICTIVA DE LA PUNTUACION EN EL TEST SOBRE EL RENDIMIENTO EN TERMODINAMICA}

Sin duda, los conceptos de calor, temperatura y energía interna son importantes en el estudio de la termodinámi-

Tabla VI

Validez predictiva de la puntuación total en el test sobre el rendimiento en termodinámica.

\begin{tabular}{|c|c|c|c|c|c|}
\hline \multirow{2}{*}{ Disciplina } & \multicolumn{2}{|c|}{$\begin{array}{l}\text { Rendimiento en } \\
\text { termodinámica }\end{array}$} & \multicolumn{2}{|c|}{$\begin{array}{c}\text { Puntuación total } \\
\text { en el test }\end{array}$} & \multirow{2}{*}{$\begin{array}{l}\text { Coeficiente de } \\
\text { correlacion entre } \\
\text { el rendimiento y la } \\
\text { puntuacion total }\end{array}$} \\
\hline & A $1 \mathrm{fa}$ & Beta & A If $\mathrm{a}$ & Beta & \\
\hline $\begin{array}{l}\text { FIS } 183(n=33) \\
\text { FIS } 183(n=30) \\
\text { FIS I } 83(n=42)\end{array}$ & $\begin{array}{l}0,84 \\
0,76 \\
0,77\end{array}$ & $\begin{array}{l}0,86 \\
0,80 \\
0,80\end{array}$ & $\begin{array}{l}0,78 \\
0,61 \\
0,78\end{array}$ & $\begin{array}{l}0,81 \\
0,67 \\
0,81\end{array}$ & $\begin{array}{l}0,59 * \\
0,69 * \\
0,46^{*}\end{array}$ \\
\hline
\end{tabular}

\footnotetext{
* Estadísticamente significativo a nivel inferior a $1 \%$.
} 
Tabia VII

Rendimiento en cada test y en la totalidad, antes y después de la disciplina.

\begin{tabular}{|l|c|c|c|c|c|}
\hline \multirow{2}{*}{ Test } & \multicolumn{2}{|c|}{$\begin{array}{c}\text { Coeficiente } \\
\text { defiabilidad }\end{array}$} & $\begin{array}{c}\text { Mediaen } \\
\text { el } \\
\text { pretest }\end{array}$ & $\begin{array}{c}\text { Media en } \\
\text { el } \\
\text { postest }\end{array}$ & $t$ \\
\cline { 2 - 6 } & A 1 f a & B cta & & & \\
\hline Calor & 0,81 & 0,83 & 3,88 & 7,23 & $6,74 \%$ \\
Temperatura & 0,70 & 0,78 & 4,00 & 4,88 & $2,01+$ \\
Energia interna & 0,37 & 0,47 & 4,29 & 4,88 & $1,78+$ \\
\hline Total & 0,81 & 0,84 & 12,21 & 16,99 & $6,79 *$ \\
\hline
\end{tabular}

is Estadísticamente significativo a njvel inferior a $1 \%$.

+ Hstadísticamente significativo a nivel inferior a $5 \%$.

ca. Es de esperar que la puntuación total en todo el test tenga algún valor predictivo sobre el desempeño de los alumnos en disciplinas de termodinámica.

En tres grupos de FIS 183 fue investigada la relación entre la puntuación total en todo el test y el promedio total de los alumnos en termodinámica. Este promedio fue obtenido a través de dos pruebas parciales de verifícación del aprendizaje. El test fue aplicado después que Ios conceptos de calor, temperatura, energía interna habían sido estudiados. Se tuvo el cuidado de investigar también los coeficientes de fiabilidad de ambas medidas (rendimiento en termodinámica y puntuación total en el test) en los tres grupos de alumnos, pues «el máximo coeficiente de correlación observable entre dos variables es menor o igual a la ráiz cuadrada de los coeficientes de fiabilidad de ambas variables" (Silveira, 1993, p. 76). La tabla VI presenta esos resultados.

Se verifica en la tabla VI que, en acuerdo con nuestra hipótesis, existe correlación entre el desempeño en la disciplina y la puntuación total en el test. Esta correlación es un argumento de validez del constructo (Silveira, 1993) para el test.

\section{SENSIBILIDAD DE LAS PUNTUACIONES TOTALES EN LA ENSENANZA DE TERMODINÁMICA}

En una muestra de 25 alumnos de FIS 183, el test fue aplicado y reaplicado antes y después de las unidades de termodinámica. La tabla VII presenta los promedios en cl pretest y postest, así como también los coeficientes alfa y beta para las puntuaciones totales.
Se observa que la única diferencia importante entre el postest y el pretest se refiere al concepto de calor. Las diferencias en las puntuaciones totales medias en temperatura y energía interna, a pesar de que estadísticamente son significativas a nivel inferior a $5 \%$ (test unicaudal), no representan cambios prácticamente relevantes (esás diferencias son menos de un acierto). Fsos resultados apuntan en el sentido de que la enseñanza de la termodinámica tiene poco o ningún efecto sobre las concepciores de los alumnos, lo cual se ha constituido en patrón reiterado en la literatura relativa a las concepciones alternativas.

\section{CONCLUSIÓN}

En este trabajo relatamos la continuidad del proceso de validación de un instrumento para verificar sí el alumno posee concepciones científicas sobre calor, temperatura y energía interna. Al mismo tiempo que describimos el proceso, colocamos a disposición del lector el test resultante, así como lo hicimos anteriormente en las áreas de mecánica y electricidad.

Pretendemos hacer algo semejante en otras áreas de la física, como, por ejemplo, en óptica. Creemos que tales instrumentos serán importantes para la práctica diariá en el aula y es por esta razón que hemos trabajado en ese sentido.

Pero, además, el presente trabajo tiene también el valor de corroborar un resultado significativo de la investigación en el área de concepciones alternativas: la poca 
influencia de la instrucción sobre el cambio conceptual. Los tests, por mejores que sean, apenas indican si el atumno posee significados científicos sobre determinado concepto, antes y después de la instrucción. El cambio conceptual no implicaría, necesartamente, el abandono de los significados alternativos sino, por lo menos,

\section{NOTAS}

* Comunicación presentada en el IV Encontro de Pesquisa em Ensino de Física, Florianópolis, del 26 al 28 de mayo de 1994 , y en el ll Simposio sobre Investigación en Enseñanza de la Física, Buenos Aires, del 4 al 6 de agosto de 1994.

\section{REFERENCIAS BIBLIOGRÁFICAS}

CRONBACH, L. J. (1951). Coefficient atpha and the internal structure of tests. Psychometrika, 16, pp. 297-334. Nueva York.

NUNNALLY, J. C. (1967). Psychometric methods. McGrawHill: Nueva York.

SILVEIRA, F, L. (1985). Coefictente beta: estimativa do coeficiente de fideơignidade de uma variável compósita. Educação e Seleçäo, 11, pp. 105-108. Säo Paulo.

SILVEIRA, F. L. (1993). Validação de testes de papel e lápis, en Moreira, M. A. y Silveira, F. L., Instrumentos de pesquisa la presencia de significados científicos en la estructura cognitiva del alumno después de la instrucción. Cuando esto no sucede, es preciso reevaluar la instrucción; los tests escritos pueden constituirse en instrumentos importantes en la evaluación de la enseñanza. Reside ahí otro de los valores de estos tests.

Los autores agradecen a Ileana Greca la tradución al español. em ensino e aprendizagem: a entrevista clínica avalidaçäo de testes de papel e läpis. Porto Alegre: EDIPUCRS.

SILVEIRA, F. L., MOREIRA, M. A. y AXT, R. (1990). Um teste para detectar concepçôs alternativas sobre calor e temperatura. Atas do $3^{\circ}$ Encontro de Pesquisa em Ensino de Física, Porto Alegre, Instituo de Física de UFRGS: 191.

SILVEIRA, F. L., MOREIRA, M. A. y AXT, R. (1991). Um teste sobre calor, temperatura e energia interna. Atas do $I X$ Simpósio Nacional de Ensino de Física, pp. 418-428. \$ão Carios.

WHERRY, R. J. (1984). Contributions to correlational analysis. Londres: Academic Press.

[Artículo recibido en noviembre de 1994 y aceptado en octubre de 1995.] 


\section{ANEXO}

Importante: No escribas nada en las hojas de preguntas. Conteste solamente esta hoja de respuestas.

\section{Nombre:}

Instruciones: A continuación, encontrarás un test constituido por 25 preguntas de elección múltiple, con tres alternativas de respuesta identificadas por los números romanos I, II y III. Puede haber una, dos o tres respuestas correctas parła cada pregunta. Señala en el gráfico la que consideres la mejor combinación de respuestas:
A. Sólo la alternativa I es correcta.
B. Sólo la alternativa II es correcta.
C. Sólo la alternativa III es correcta.
D. I as alternativas I y II son correctas.
E. L.as alternativas I y III son correctas.
F. Las altenativas II y III son correctas.
G. Todas las alternativas son correctas.

\begin{tabular}{|c|c|c|c|c|c|c|c|}
\hline & A & B & C & D & F & F & G \\
\hline 1 & & & & & & & \\
\hline 2 & & & & & & & \\
\hline 3 & & & & & & & \\
\hline 4 & & & & & & & \\
\hline 5 & & & & & & & \\
\hline 6 & & & & & & & \\
\hline 7 & & & & & & & \\
\hline 8 & & & & & & & \\
\hline 9 & & & & & & & \\
\hline 10 & & & & & & & \\
\hline 11 & & & & & & & \\
\hline 12 & & & & & & & \\
\hline 13 & & & & & & & \\
\hline
\end{tabular}

\begin{tabular}{|c|c|c|c|c|c|c|c|}
\hline & A & B & C & D & E & F & G \\
\hline 14 & & & & & & & \\
\hline 15 & & & & & & & \\
\hline 16 & & & & & & & \\
\hline 17 & & & & & & & \\
\hline 18 & & & & & & & \\
\hline 19 & & & & & & & \\
\hline 20 & & & & & & & \\
\hline 21 & & & & & & & \\
\hline 22 & & & & & & & \\
\hline 23 & & & & & & & \\
\hline 24 & & & & & & & \\
\hline 25 & & & & & & & \\
\hline
\end{tabular}

Fecha: 

1. Asociamos la existencia de calor:
1) a cualquier cuerpo, pues todo cuerpo posee calor;
II) sólo a aquellos cuerpos que están «calientes»;
III) a sifuaciones en las cuales ocurre, necesariamente, transferencia de energía.

2. Para que se pueda hablar de calor:

1) es suficiente un unico sistema (cuerpo);

II) son necesarios, por lo menos, dos sistemas;

III) es suficiente un único sistema, pero tiene que estar «caliente».

3. Para que se pueda admitir la existencia de calor debe haber:

I) una diferencia de temperaturas;

II) una diferencia de masas;

III) una diferencia de energías.
4. Calor es:
I) energía cinética de las moléculas;
II) energfa transmitida sólo por medio de una diferencia de temperaturas;
III) la energía contenida en un cuerpo.
5. En el interior de una habitación que no haya sido calentada o refrigerada durante varios días:
1) la temperatura de los objetos de metal es inferior a la temperatura de los objetos de madera;
II) la temperatura de los objetos de metal, de las mantas y de los demás objetos es la misma;
III) ningún objeto presenta temperatura.
6. El agua (a $\mathrm{O}^{\circ} \mathrm{C}$ ) que resulta de la fusión de un cubito de hielo (a $\mathrm{O}^{\circ} \mathrm{C}$ ), contiene, respecto al hielo:
I) más energía;
II) menos energía;
III) igual cantidad de energía.

7. Una mezcla de hielo y agua, ambos a $\mathbf{O}^{\circ} \mathrm{C}$, se matiene a esa temperatura. En esas condiciones:

I) se funde todo el hielo;

II) se funde parte del hiejo;

III) no se funde el hielo. (ítem eliminado)

8. Dos cubos metálicos A y B son colocados en contacto. A está más «caliente» que B. Ambos están más «calientes» que el ambiente. Al cabo de un cierto tiempo la temperatura final de A y B será:

I) igual a la temperatura ambiente;

II) igual a la temperatura inicial de B;

III) un promedio entre las temperaturas iniciales de $\mathbf{A}$ y $\mathbf{B}$.

9. Dos pequeñas placas A y B del mismo metal y del mismo espesor son colocadas en el interior de un horno, el cual es cerrado y luego accionado. La masa de $A$ es el doble de la masa de $B\left(m_{A}=2 m_{a}\right)$. Inicialmente las placas y el horno están todos a la misma temperatura. Algún tiempo después la temperatura de $A$ será: 
I) el doble de la de $\mathrm{B}$;
II) la mitad de la de $\mathrm{B}$;
III) igual a la de $\mathrm{B}$.

10. Considere dos esferas idénticas, una en un horno caliente y la otra en un congelador. Básicamente, ¿qué diferencia hay entre ellas inmediantamente después de sacarlas del horno y de la heladera respectivamente?

l) La cantidad de calor contenida en cada una de elias.

II) La temperatura de cada una de ellas.

III) Una de cllas contiene calor y la otra no.

11. En dos vasos idénticos que contienen la misma cantidad de agua (aproximadamente $250 \mathrm{~cm}^{3}$ ) a temperatura ambiente son colocados un cubito de hielo a $0^{\circ} \mathrm{C}$ y tres cubitos de hielo a $\mathrm{O}^{\circ} \mathrm{C}$ respectivamente (cada cubito con aproximadamente $\left.1 \mathrm{~cm}^{3}\right)$. ¿En cuál situación el agua se enfría más?

I) En el vaso donde son colocados tres cubitos de hielo.

II) En el vaso donde es colocado un cubito de hielo.

III) Se enfría igualmente en los dos vasos.

12. Dos esferas del mismo material pero cuyas masas son diferentes quedan durante mucho tiempo en un horno. Al retirarlas del horno, son inmediatamente puestas en contacto. En esa situación:

I) fluye calor de la esfera de mayor masa hacia la menor masa;

II) fuye calor de la esfera de menor masa hacia la mayor masa;

III) ninguna de las dos esferas cede calor a la otra.

13. I as mismas esferas de la pregunta anterior son ahora dejadas durante mucho tiempo en un congelador. En esa situación, al retirarlas e inmediatamente ponerlas en contacto:

I) ninguna de las esferas posee calor debido a su baja temperatura;

II) fluye calor de la esfera de mayor masa hacia la de menor masa;

III) ninguna de las esferas puede ceder calor a la otra.

14. ¿Qué cambia cuando una cantidad de agua que ya está hirviendo pasa, por ebullición, a estado de vapor?

I) Su energía interna.

II) EI calor contenido en ella.

III) Su temperatura.

15. Cuando las extremidades de una barra metálica están a temperaturas diferentes:

I) la extremidad a mayor temperatura tiene más calor que la otra;

II) el calor fluye de la extremidad que contiene más calor hacia la que contiene menos calor;

III) existe transferencia de energía por el movimiento desordenado de átomos o moléculas.

16. I,a energía interna de un cutrpo puede ser asociada con:

I) calor;

II) energia cinética de átomos o moléculas;

III) energías potenciales de átomos o moléculas. 


\section{Completa la siguiente frase:}

La elevación de temperatura que percibes cuando frotas tus manos es resultado de conducción de hacia el interior de las manos. Resulta, en virtud de ello, un aumento de sa

I) trabajo, całor, energía interna;

II) calor, energía, temperatura;

III) trabajo, temperatura, calor. (ítem eliminado)

18. Si se observa la figura sin disponer de ninguna ofra información, se puede decir que el cubo A posee, respecto al ambiente que lo circunda:

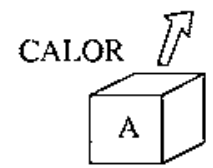
I) temperatura más elcvada;
II) más energía;
III) más calor.

19. Cuando se encuentra a la presión atmosférica, el nitrógeno líquido entra en ebullición a $-196^{\circ} \mathrm{C}$. Un gramo de nitrógeno líquido, a esa temperatura, comparado con un gramo de vapor de nitrógeno, también a $-196^{\circ} \mathrm{C}$, posee:
1) más energía;
II) menos energia;
III) igtual cantidad de energía.

20. Fi punto de solidificación del mercurio, a la presión atmosférica, es $-39^{\circ} \mathrm{C} . \dot{\text { qu }}$ Qué pasa inmediatamente después de que una cierta cantidad de mercurio líquido (a $-39^{\circ} \mathrm{C}$ ) es colocada en nitrógeno líquido (a $\left.-196^{\circ} \mathrm{C}\right)$ ?

I) La temperatura del nitrógeno aumenta y la del mercurio disminuye.

II) La temperatura del mercurio disminuye pero la del nitrógeno no se altera.

III) El mercurio comienza a solidificar y el nitrógeno entra en ebullición, sin cambio en la temperatura.

21. ¿Qué sucede cuando colocamos un termómetro, en un día de temperatura ambiente igual a 21 "C, en agua a una temperatura más elevada?

I) La temperatura y la energía interna del termómetro aumentan.

I) La temperatura de termómetro aumenta pero su energía interna permanece constante.

II) Ni la temperatura del termómetro ni su energía interna se modifican, sólo la columna del líquido termométrico se dilata.

22. Cuando, con el mismo ebullidor, se calientan $100 \mathrm{mI}$ de agua y $100 \mathrm{mI}$ de alcohol, es posible constatar que el tiempo necesario para elevar $1^{\circ} \mathrm{C}$ la temperatura de $1 \mathrm{~g}$ de agua es mayor que el tiempo necesario para que ocurra lo mismo con 1 gr de alcohol. Esto significa que el agua acumula, en comparación con el alcohol:

1) la misma cantidad de energía;

II) más energía;

III) menos energía. 
23. Observa la figura y considera el cuerpo $\mathrm{C}$ (sombreado) un conductor de calor. ¿Qué caracteriza esta situación de conducción de calor?

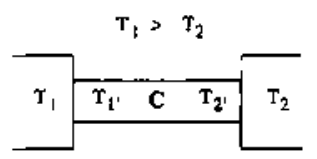
I) $T_{1}^{\prime}=T_{2}^{\prime} ;$
II) $\mathrm{T}_{1}{ }^{\prime}>\mathrm{T}_{2}{ }^{\prime}$;
III) $T_{1}^{\prime}<T_{2}^{\prime}$.

24. Cuando un buen conductor es colocado en contacto con otro cuerpo cuya temperatura es más alta, el conductor transfiere energía:
1) sin modificar su temperaturá;
II) modificando su temperatura;
III) modificando su energía interna.

25 . Objetos de metal y de material plástico son puestos en el interior de un congelador que se encuentra a $-20^{\circ} \mathrm{C}$. Después de algunos días se puede afirmar que la temperatura de los objetos de plástico es:
I) mayor que la temperatura de los objetos de metali
II) menor que la temperatura de los objetos de metal;
III) igual a la temperatura de los objetos de metal. 